\title{
Tomographic Separation of Composite Spectra of O-Type Stars
}

\author{
W. G. BAGNUOLO \& D. R. GIES \\ CHARA, Georgia State University, Atlanta, GA 30303, USA
}

\begin{abstract}
We have analyzed UV photospheric lines of three O-type binaries (AO Cas, Plaskett's Star, and $29 \mathrm{UW} \mathrm{CMa)} \mathrm{by} \mathrm{means} \mathrm{of} \mathrm{tomographic} \mathrm{and} \mathrm{cross-}$ correlation analyses, with the goal of estimating the physical properties of the individual stars. The tomographic techniques allowed us to estimate $T_{e f f}$ and $\log g$, the luminosity ratio, and rotational velocities. The mass ratios were obtained from a cross-correlation technique, applied to spectral regions in which preliminary tomography shows the secondary has strong photospheric line features.
\end{abstract}

\section{ALGORITHMS AND DATA REDUCTION}

Ultraviolet spectra with good orbital phase coverage were gathered from the IUE Regional Data Analysis Facility in Boulder. Although these data were used initially to investigate the stellar winds, they do cover about $80 \%$ of the spectrum from $1340-1780 \AA$. We used 16,17 , and 28 data sets for AO Cas, Plaskett's Star, and 29 UW CMa respectively.

The tomography algorithm used was a version of the ILST (Iterative LeastSquares Technique). For a binary star, we can imagine a luminous 'object' two pixels thick by $n$ pixels long, where $n$ is the number of pixels per spectrum. The radial velocity differences of the stars at different epochs correspond to different view angles for tomography. See Bagnuolo \& Gies 1991 (BG) for details.

\section{AO CASSIOPEIAE}

AO Cas is a 3.52d double-lined spectroscopic binary (O9 III, Walborn 1973). Historically, mass ratios have been derived ranging from 1.0 to 1.4 , mainly by modelling of the ellipsoidal light curve. The temperatures are relatively pooly determined, but it was thought that the primary is slightly hotter.

Our results for this star have been presented elsewhere (BG). Table 1 summarizes the physical parameters found for both components of this star, as well as for the other two stars; one basic result is that the secondary is slightly hotter. Figures $1 \mathrm{a}$ and $1 \mathrm{~b}$ show that the secondary (lower spectra, both spectra rectified to unit continuum) has stronger $\mathrm{Fe} \mathrm{V}$ lines than the primary (upper spectra), but weaker Fe IV lines.

\section{PLASKETT'S STAR}

Plaskett's Star (HD 47129) has generally been considered the most massive binary since its discovery in 1922 . The spectral type of the primary was estimated variously as O7.5 III(f) by Garmany et al. (1980) and O7 I by Hutchings $\&$ Cowley (1976). The secondary has been an enigma and secondary lines had not been previously found in IUE data. Because of the poor quality of secondary 
TABLE 1. Derived Stellar Properties

\begin{tabular}{|c|c|c|c|c|c|c|}
\hline \multirow[t]{2}{*}{ Parameter } & \multicolumn{2}{|c|}{ AO Cas } & \multicolumn{2}{|c|}{ Plaskett's Star } & \multicolumn{2}{|c|}{$29 \mathrm{UW} \mathrm{CMa}$} \\
\hline & Primary & Secondary & Primary & Secondary & Primary & Secondary \\
\hline $\begin{array}{l}M / \mathcal{M}_{\odot} \\
\text { Int. Sp. Cless } \\
T_{\text {eff }} \\
\log L / L_{\odot} \\
R / R_{\odot} \\
\log g\end{array}$ & $\begin{array}{c}12.4 \\
\text { O9.5 I } \\
32000 \\
5.06 \\
10.9 \\
3.29\end{array}$ & $\begin{array}{c}18.3 \\
08.1 \mathrm{~V} \\
36000 \\
4.82 \\
6.3 \\
4.00\end{array}$ & $\begin{array}{c}42.5 \\
\text { O7.3 I } \\
35100 \\
5.80 \\
21.5 \\
3.4\end{array}$ & $\begin{array}{c}51.0 \\
\text { O6.2 I } \\
38400 \\
5.57-5.94 \\
13.8-21.1 \\
3.5-3.9\end{array}$ & $\begin{array}{c}20.0 \\
\text { OB I } \\
33000 \\
5.98 \\
30 \\
2.8\end{array}$ & $\begin{array}{c}22.4 \\
\text { B0 I } \\
26000 \\
5.41 \\
25 \\
3.0\end{array}$ \\
\hline$V \sin i\left(\mathrm{~km} \mathrm{~s}^{-1}\right)$ & 120 & 130 & 75 & 310 & - & - \\
\hline
\end{tabular}
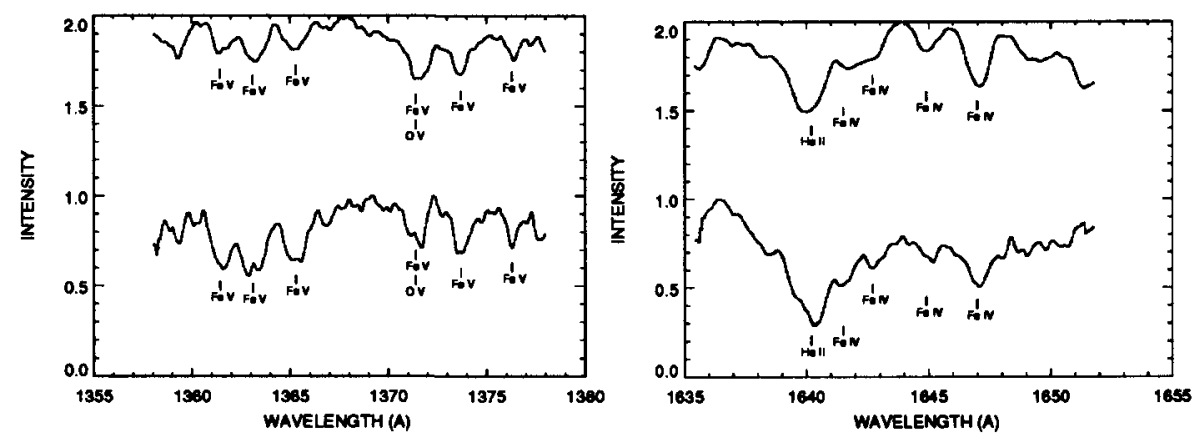

FIGURE 1 (above). Separated spectra of AO Cassiopeiae FIGURE 2 (below). RV curve and separated spectra of Plaskett's Star
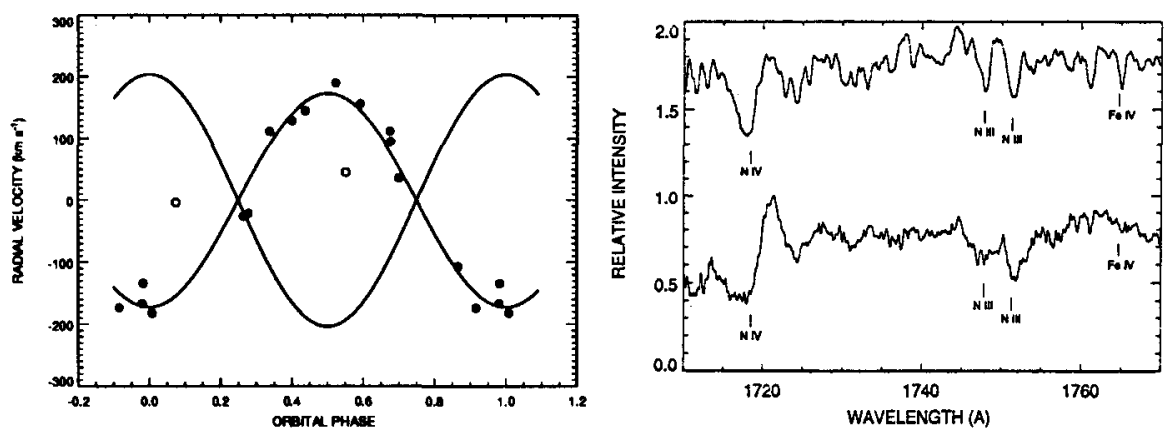

orbital data, the mass ratio and masses are uncertain.

We performed a preliminary tomographic analysis (using the primary orbit by Stickland 1987) and identified the three regions of the strongest secondary lines. We then did a cross-correlation analysis of these regions, producing the radial velocity curve for the secondary shown in Figure 2a, allowing estimates of the masses. (See also Bagnoulo et al. 1992) The final tomographic analysis produced sets of reconstructed spectra of which an example is shown in Figure $2 \mathrm{~b}$. Note the rotationally broadened lines of the secondary (bottom spectra) and the relative weakness of the secondary Fe IV lines. The secondary has a 
characteristic P Cygni profile for the N IV $\lambda 1718$ line compared to the primary, indicating that the former has a somewhat stronger wind.

The most unexpected results of these analyses (see Table 1) were the earlier type and rapid rotation of the secondary. Two evolutionary scenarios suggest themselves. In the first, both primary and secondary are core hydrogen burning objects evolving off the main sequence. The evolution of the more massive secondary has been retarded by its rapid rotation. The second is an Algol-type system. The primary was initially more massive, but evolved to "red point" on the evolutionary track, filling its Roche lobe and transferring mass and angular momentum to the secondary. The secondary was spun up the large rotational velocity we currently observe. We favor the second scenario for several reasons. If so, there should be a number of 'Plaskett-type' systems, in which the initial separation is close enough to allow a Roche lobe overflow, but far enough to allow the secondary to remain 'spun up' without complete tidal braking.

\section{29 UW CMA}

Finally, the secondary of $29 \mathrm{UW}$ CMa had not been seen in the UV, and estimated mass ratios range from $q=0.75$ (Leung \& Schneider 1978) to $q=1.30$ (Hutchings 1977). We determined the mass ratio to be $q=1.12$, using techniques as in BG. The secondary is clearly later in type (Fe V lines are weak), but also has several spectral pecularities including the unusually strong $\lambda 1723$ blend. The primary wind appears to completely dominate, unlike for the other two stars. Figures $3 \mathrm{a}$ and $3 \mathrm{~b}$ show two separated spectra for this star.
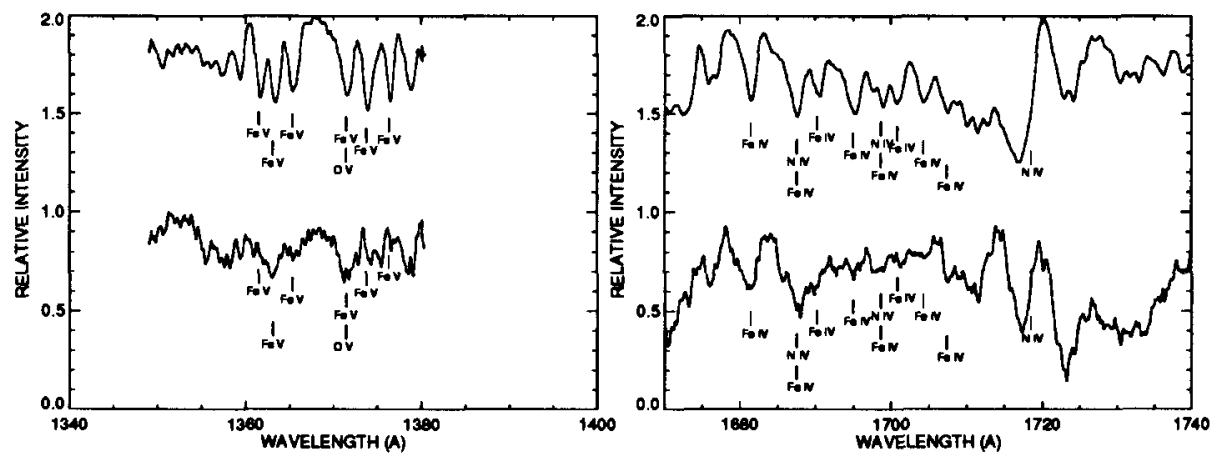

FIGURE 3. Separated spectra of UW CMa

\section{REFERENCES}

Bagnuolo, W.G. Jr. \& Gies, D.R. 1992, $A p J, 376,266$

Bagnuolo, W.G. Jr., Gies, D.R., \& Wiggs, M.S. 1992, ApJ, 385, 708

Garmany, C.D., Conti, P.S., \& Massey, P. 1980, ApJ, 242, 1063

Hutchings, J.B. 1977, PASP, 89, 668

Leung, K.C. \& Schneider, D.P. 1978, ApJ, 222, 924

Stickland, D.J. 1987, Observatory, 107, 68

Walborn, N.R. 1973, $A J, 78,1067$ 